\title{
Selected papers of the Third International Conference on the Theory and Practice of Natural Computing, TPNC 2014
}

\author{
Adrian-Horia Dediu ${ }^{1}$. Carlos Martín-Vide ${ }^{2}$
}

Published online: 18 September 2017

(C) Springer-Verlag GmbH Germany 2017

This special issue of the journal 'Soft Computing—A Fusion of Foundations, Methodologies and Applications' offers extended versions of some of the best papers presented at the Third International Conference on the Theory and Practice of Natural Computing, TPNC 2014, held in Granada, Spain, on December 9-11, 2014, under the organization of the Research Group on Mathematical Linguistics (GRLMC) from Rovira i Virgili University in Tarragona, Spain, and the Soft Computing and Intelligent Information Systems research group $\left(\mathrm{SCI}^{2} \mathrm{~S}\right)$, from University of Granada, Spain.

TPNC 2014 was the third event in a series dedicated to host and promote research in a wide spectrum of computational models, methods, and techniques inspired by information processing in nature. We encourage both theoretical contributions in soft computing, computing architectures, and formal models and solutions for practical problems based on natural computing methods.

Out of 47 submissions to the conference, 22 papers were accepted (which represents a competitive acceptance rate of about $47 \%$ ). Among them, the authors of 11 papers were invited to submit to this special issue. Each submission was reviewed by at least two experts, and on the basis of their comments, the guest editors decided to accept six papers for this special issue (which represents an acceptance rate of about $13 \%$ out of the submissions to the conference).

We regret that Rūsinsš Freivalds sadly passed away before finishing the revision of his submitted single-authored paper,

Adrian-Horia Dediu

adrian-horia.dediu@superdata.ro

1 SuperData, Bucharest, Romania

2 Research Group on Mathematical Linguistics (GRLMC), Rovira i Virgili University, Av. Catalunya, 35, 43002

Tarragona, Spain which thus could not be considered for this special issue any more.

Next, we present the papers included in our special issue sorted by the first author name. For each paper, we mention the most important results.

Henning Bordihn, Paolo Bottoni, Anna Labella, and Victor Mitrana, in their paper Networks of Picture Processors as Problem Solvers, show how to use networks of picture processors (NPP) for pattern matching. Network processors performing insertion, deletion, and substitution of symbols in the pictures in the two-dimensional plane have been investigated earlier by the authors for various theoretical properties such as their computational power. We note that suitable additional features like new operations on pictures, called "mask" (which changes visible symbols into invisible copies) and "unmask" (the reverse operation), lead to networks with a different kind of nodes, called hiding picture processors, thereby yielding efficient solutions for finding a pattern in an input picture, with the pattern being a fixed picture.

In their paper, Extrapolated Quantum States, Void States, and a Huge Novel Class of Distillable Entangled States, Michel Boyer, Aharon Brodutch, and Tal Mor introduce and extensively study the notions of boundary-separable states, epsilon-entangled states, boundary-classical states, and epsilon-discordant states. The results are based on many interesting families of states, and many novel constructions are followed by suggestive examples. The discussion on twoqubit states for higher dimensions is particularly important because many facts about entanglement are dependent on the subspace dimensions.

José-Matías Cutillas-Lozano and Domingo Giménez propose a methodology for auto-tuning the parameters of a parallel message-passing model in their paper Optimizing a Parameterized Message-passing Metaheuristic Scheme on a Heterogeneous Cluster. Starting from a previous shared 
memory approach, the authors introduce and analyze the influence of several new parameters such as migration size and frequency and the number of used processes. To validate the new method, the paper presents, as an example, how to find an optimal solution to a problem of electricity consumption in an exploitation of wells. The results show that the message-passing scheme is faster than the shared memory one on a homogeneous system.

A survey paper, Since CEC 2005 Competition on Realparameter Optimisation: A Decade of Research, Progress and Comparative Analysis's Weakness by Carlos GarcíaMartínez, Pablo David Gutiérrez, Daniel Molina, Manuel Lozano, and Francisco Herrera, tries to analyze the progress of the algorithms in the field of real-coding optimization. The authors discuss about several existent benchmarks pointing out that their adoption in algorithm comparisons is limited and thus the real progress of some new methods is difficult to analyze. The paper concludes that, despite the exponential growth of the published results, the progress in the field is rather slow.

Mohammad Ali Javaheri Javid, Wajdi Alghamdi, Anna Ursyn, Robert Zimmer, and Mohammad Majid al-Rifaie, in their paper Swarmic Approach for Symmetry Detection of Cellular Automata Behaviour, use a swarm intelligence
algorithm-Stochastic Diffusion Search—in detecting symmetries on grid lattices of cellular automata. A contextsensitive mechanism together with a partial function evaluation is the main ingredient to deal with the complexity issues. The results show the good performance of the algorithm used.

In the paper entitled Using Mixed Mode Programming to Parallelize an Indicator-Based Evolutionary Algorithm for Inferring Multiobjective Phylogenetic Histories by Sergio Santander-Jiménez and Miguel A. Vega-Rodríguez, the authors present a parallel implementation to solve the reconstruction of phylogenetic trees. The main contribution of the article is the use of a shared hybrid distributed memory system to improve the multiobjective parallel search capabilities of the implementation.

We thank all reviewers for their valuable work. We also thank all the authors for their contributions as well as the editorial team of the journal for their professional support and collaboration.

\section{Compliance with ethical standards}

Conflict of interest The authors declare that they have no conflict of interest. 